Tina Freyburg

\title{
Wenn die Wüste blüht
}

\author{
Eine Replik auf Thomas Bräuninger
}

In meinem ZIB-Aufsatz "Demokratisierung durch Zusammenarbeit?" präsentierte ich Ergebnisse meiner Studie zum demokratisierenden Potenzial transgouvernementaler Politiknetzwerke, die Staatsbeamte aus etablierten Demokratien und nichtdemokratischen Regimen auf der Verwaltungsebene zur Lösung politikfeldspezifischer Probleme zusammenbringen. Konkret erörterte ich die Frage, inwieweit und inwiefern Twinning-Projekte der Europäischen Union die Einstellungen von involvierten Staatsbeamten zu demokratischem Regieren in stabilen autoritären Regimen wie Marokko positiv prägen. Die Ergebnisse multipler Querschnittsregressionsanalysen auf Basis originärer Umfragedaten zeigen, dass transgouvernementale Netzwerke, unter der Bedingung eines vorherigen Aufenthaltes im demokratischen Ausland, Staatsbeamte in autoritären Regimen in demokratischem Regieren sozialisieren können. In seiner Replik weist Thomas Bräuninger zu Recht auf einige methodische Herausforderungen der Sozialisierungsforschung hin. Auch nach einer wiederholten Analyse meiner Daten im Lichte seiner Bedenken und Vorschläge finde ich ausreichend empirische Evidenz für ein demokratisierendes Potenzial funktionaler Kooperation in transgouvernementalen Netzwerken.

\section{Vorbemerkung}

In meinem 2011 in der ZIB erschienenen Aufsatz »Demokratisierung durch Zusammenarbeit? « (Freyburg 2011) präsentierte ich Ergebnisse meiner Studie zum demokratisierenden Potenzial transgouvernementaler Politiknetzwerke, die Staatsbeamte aus etablierten Demokratien und stabilen autoritären Regimen auf der Verwaltungsebene zur Lösung politikfeldspezifischer Probleme zusammenbringen. ${ }^{1}$ Meine Studie legte damit empirische Evidenz für das bereits seit Längerem von Politikwissenschaftlern $^{2}$ wie Anne-Marie Slaughter (2004), Robert O. Keohane/Joseph S. Nye (1974) und Kai Raustiala (2002) postulierte Sozialisierungspotenzial transgouvernementaler Netzwerke vor. Mit dem Fokus auf die demokratisierende Wirkung von Verwaltungskooperationen zeigte meine Arbeit zudem alternative innovative Ziele und Wege für die Förderung demokratischer Prinzipien und Praktiken von außen auf. Damit verbindet mein Beitrag, in den Worten von Thomas Bräuninger

1 Ich danke Fabrizio Gilardi, Philipp Rehm, Solveig Richter, Rebecca Welge, Julian Wucherpfennig und vor allem Tobias Böhmelt für ihre hilfreichen Anmerkungen zu einer früheren Version. Etwaige Missverständnisse und Fehler liegen allein in meiner Verantwortung.

2 Ich verwende im Folgenden aus Gründen der Einfachheit die männliche Form. 
(2012: 85, in diesem Heft), »in geradezu idealer Weise politik-wissenschaftliche und politisch-praktische Relevanz«.

Konkret argumentierte ich in meinem Beitrag, dass transgouvernementale Netzwerke Beamten in nichtdemokratischen Ländern die Möglichkeit bieten, mit demokratischen Prinzipien und Praktiken administrativen Regierens vertraut zu werden. Ich überprüfte dieses Argument empirisch am Beispiel von vier Twinning-Projekten, welche die Europäische Union (EU) in den Politikbereichen Gesundheitskontrolle, Umwelt, Wettbewerb und Zölle in marokkanischen Ministerien implementierte. Um den Effekt der Teilnahme an einem Twinning-Projekt auf die Einstellungen zu demokratischem Regieren zu schätzen, wäre eine Längsschnittanalyse der Einstellungen der betroffenen Beamten vor und nach ihrer Teilnahme ideal gewesen. Eine solche Panelstudie war leider im Rahmen dieser Studie - wie auch von Bräuninger angemerkt - nicht realisierbar. Aus den in meinem Beitrag genannten Gründen führte ich alternativ eine Querschnittsstudie durch, in der ich die Einstellungen teilnehmender Beamter mit den Einstellungen nicht-teilnehmender Kollegen bei gleichzeitiger Kontrolle relevanter alternativer Erklärungsfaktoren verglich. Ein solches Design hat selbstverständlich seine Stärken und Grenzen, wie von mir in meinem Aufsatz sowie von Thomas Bräuninger in seiner Replik problematisiert. Ich danke Thomas Bräuninger für sein Interesse an meiner Arbeit, seine sorgfältige Replikation meiner Analysen und seine wohlüberlegten Vorschläge alternativer Herangehensweisen zur Lösung des Problems der kausalen Inferenz.

\section{Replikation und Ergänzung meiner Studie im Lichte Bräuningers Anmerkungen}

Zum besseren Verständnis des vorliegenden Austausches möchte ich die zentralen Hypothesen meines Beitrages kurz in Erinnerung rufen (Freyburg 2011: 12-13):

H1: Staatsbeamte, welche in transgouvernementale Politiknetzwerke involviert waren, habe[n] eine positivere Einstellung zu demokratischem Regieren als ihre Kollegen, die nicht an diesen Netzwerken teilnahmen (»Kooperation«).

Es ist wahrscheinlicher, dass die Teilnahme an transgouvernementalen Netzwerken einen positiven Einfluss auf die Einstellungen zu demokratischem Regieren von Staatsbeamten ausübt, wenn diese...

$H 2 a$... längere Zeit in einer westlichen Demokratie zu Ausbildungs- oder beruflichen Zwecken verbracht haben (Auslandsaufenthalt);

$H 2 b . .$. regelmäßig westliche Medien zur politischen Information nutzen (ausländische Medien).

Ich untersuchte diese Hypothesen anhand einer Reihe von multiplen Querschnittsregressionsanalysen auf Basis originärer Umfragedaten, die ich mithilfe einer Befragung von 150 marokkanischen Staatsbeamten zu ihren Einstellungen zu demokratischem Regieren im Sommer 2008 selbst erhoben hatte. Die Interpretation der Regressionsergebnisse stützte ich durch einen qualitativen Vergleich des sozialis- 
ierenden Potenzials der einzelnen Projekte sowie durch Erkenntnisse ab, die ich aus qualitativen Interviews mit Staatsbeamten aus Marokko und ihren europäischen Kooperationspartnern gewann. In den empirischen Daten finde ich keinen Nachweis für die Hypothesen 1 und 2b, wohl aber für Hypothese 2a (Freyburg 2011: 24-25). Im Gegensatz dazu argumentiert Thomas Bräuninger (2012: 87), dass aus drei Gründen »die zentralen Ergebnisse der empirischen Analyse« die Haupthypothesen inklusive Hypothese 2a nicht stützen. Erstens, Bräuninger vermutet ein etwaiges Selektionsproblem bei der Auswahl der Staatsbeamten und ihrer Zuweisung zu Kontroll- bzw. Fokusgruppe, so dass potenzielle konfundierende Faktoren die Ergebnisse der empirischen Analyse verzerren könnten. Zweitens, hinsichtlich des von mir erwarteten begünstigenden Effekts alternativer transnationaler Einflussfaktoren auf die hypothesierte demokratisierende Wirkung transgouvernementaler Netzwerke bringt Bräuninger zwei kritische Einwände vor. Zum einen gewann er den Eindruck einer fehlerhaften theoretischen Herleitung des erwarteten Interaktionseffektes. Zum anderen empfiehlt er eine alternative Interpretation des statistischen Interaktionsterms, welche eine Zurückweisung des postulierten bedingten Sozialisierungseffektes nahelegt. Drittens, eine wiederholte Analyse des potenziellen demokratisierenden Effektes der einzelnen Projekte bestätigt Bräuninger darin, die von mir postulierte demokratisierende Wirkung transgouvernementaler Netzwerke zurückzuweisen. Im Folgenden werde ich auf diese drei Hauptpunkte Bräuningers eingehen und sie angemessen diskutieren. Auch nach einer wiederholten Analyse meiner Daten im Lichte seiner Bedenken und Vorschläge finde ich ausreichend empirische Evidenz für eine Bestätigung der Hypothese 2a.

\subsection{Selektion der Staatsbeamten und ihre Zuweisung zur Fokus- bzw. Kontroll- gruppe}

Um den Effekt der Teilnahme an einem Twinning-Projekt auf die Einstellung zu demokratischem Regieren zu schätzen, vergleiche ich die Einstellungen der Beamten, die an einem solchen Projekt teilnahmen (»Fokusgruppe«) mit den Einstellungen von Beamten, die nicht an einem Twinning-Projekt teilnahmen (»Kontrollgruppe«) mithilfe statistischer Verfahren. »Die Differenz in der Einstellung zu demokratischem Regieren zwischen diesen beiden Gruppen wird dem Effekt der Teilnahme an Twinning-Projekten zugeschrieben, bei gleichzeitiger expliziter Kontrolle des möglichen Einflusses anderer relevanter Eigenschaften der Staatsbeamten (quasi-experimenteller statischer Gruppenvergleich)« (Freyburg 2011: 17; vgl. Campbell/Stanley 1966: 12; Halaby 2004: 509-12). Wie Bräuninger zu Recht anmerkt, ist eine solche kausale Inferenz problematisch und nur unter zwei Bedingungen zulässig. Es muss sichergestellt werden, dass, erstens, die Zuweisung der Beamten zu Kontroll- und Fokusgruppe nicht auf bestimmten Faktoren beruht, die nicht in die Analyse eingehen, und, zweitens, die beiden erhaltenen Gruppen in allen als relevant erachteten Charakteristika ähnlich sind. Nur wenn »sich die Individuen in beiden Gruppen im Durchschnitt und bei hinreichend großer Fallzahl nicht wesentlich 
hinsichtlich dritter, möglicherweise konfundierender Eigenschaften unterscheiden [,... kann] der Unterschied in der abhängigen Variablen zuverlässig mit dem Treatmenteffekt in Verbindung gebracht werden« (Bräuninger 2012: 89).

Grundsätzlich kann diese Problematik mithilfe zweier Verfahren adressiert werden: die Zufallsstichprobe im Vorfeld der Befragung oder das matching der beiden Gruppen im Nachhinein. Da es nicht möglich war, umfassende und zuverlässige Informationen über die Gesamtpopulation der Staatsbeamten in Marokko zu erhalten, welche für eine ausreichend repräsentative Zufallsauswahl notwendig ist, wählte ich ein »theoretisch kontrolliertes Clusterverfahren« (Freyburg 2011: 17). Dieses Verfahren ist nützlich "when a complete listing of the population is not available« (Agresti/Finlay 2009: 23). Entsprechend dieses Verfahrens identifizierte ich zuerst die Abteilungen in verschiedenen Ministerien (cluster), die an einem Twinning-Projekt teilnahmen. Das Projekt Unterstützung der Stärkung der Wettbewerbsbehörden (MA06/AA/FI08) zum Beispiel begünstigte die Abteilung für Wettbewerb und Preise des marokkanischen Ministeriums für wirtschaftliche Angelegenheiten. Anschließend wählte ich thematisch ähnliche Abteilungen, die an einem solchen Projekt nicht teilnahmen. Eine geeignete Kontrollgruppe für die Wettbewerbsabteilung ist beispielsweise die Abteilung für Staatsvermögen und externe Finanzen des Ministeriums für Wirtschaft und Außenfinanzierung. Alle Beamten in den so ausgewählten Abteilungen bildeten das Sample dieser Studie. Dieses in der Sozialisationsforschung übliche Verfahren (vgl. Pollack 1998: 10-11) birgt das Risiko einer Selbstselektion bzw. einer selektiven Rekrutierung. Bereits 1973 machte Kerr (1973) in seinem Aufsatz über den Einstellungswandel von Mitgliedern des Europäischen Parlamentes zum Integrationsprojekt darauf aufmerksam, dass vor allem EU-freundliche Parlamentarier bereit wären, nach Brüssel zu gehen und der gefundene Sozialisationseffekt damit eher ein nationaler als ein internationaler wäre. Für meine Studie war daher von Bedeutung zu überprüfen, ob beispielsweise nur besonders regimetreue oder europafreundliche Staatsbeamte für die Teilnahme an einem Twinning-Projekt ausgewählt wurden. In diesem Fall hätten bereits vorab existierende Attribute einen möglichen sozialisierenden Effekt der Netzwerke negativ bzw. positiv beeinflussen können. Ein solcher Selektionseffekt konnte für diese Studie aus zwei Gründen vernachlässigt werden. Erstens, alle Beamten, die für eine begünstigte Abteilung arbeiten, gehören prinzipiell zur Zielgruppe eines TwinningProjektes (Freyburg 2011: 15). Zweitens, alle Beamten, die in den ausgewählten Abteilungen arbeiten, wurden gebeten, den Fragebogen auszufüllen; die Rücklaufquote betrug etwa 96\% (Freyburg 2011: 17). Damit erfolgte die Auswahl der befragten Beamten und ihre Zuweisung zu Fokus- bzw. Kontrollgruppe nicht aufgrund bestimmter Personencharakteristika, sondern allein auf Grundlage der Kernvariable meiner Studie: (Nicht-)Teilnahme an einem Twinning-Projekt. ${ }^{3}$

3 Auf die Frage eines etwaigen Selektionsbias hinsichtlich der alternativen Erklärungsvariable »Auslandsaufenthalt« gehe ich im nächsten Abschnitt zur Interpretation des Interaktionseffektes ein. 
Inwieweit sind die erhaltenen beiden Gruppen ähnlich hinsichtlich relevanter Personencharakteristika? Zur Überprüfung dieser zweiten Bedingung greife ich Bräuningers sehr guten Vorschlag eines matching der Fokus- und Kontrollgruppe auf. Nicht-parametrische matching-Methoden erlauben Beobachtungsdaten nachträglich so zu vergleichen, dass sie in etwa Experimentaldaten entsprechen, also die Fokus- und Kontrollgruppen »in allen als relevant erachteten Charakteristika ähnlich sind« (Bräuninger 2012: 90). Gegenüber einer einfachen Regressionsanalyse hat dieses Verfahren den Vorteil, dass es für die Effekte einer etwaigen systematischen Selektion auf den beobachteten Variablen unabhängig deren funktionaler Form kontrolliert. Zur Berücksichtigung möglicher Konfundierungseffekte replizierte ich die Analysen mittels eines genetischen Eins-zu-Eins-matching mit Ersetzen. Nach dieser Methode werden Beobachtungen auf der Basis relevanter Kovariaten ausgewählt, um die Balance - $\gg$ the degree to which the treatment and control covariate distributions resemble each other« (Ho et al. 2007: 217) - zu erhöhen. Bei den Kovariaten handelt es sich neben zwei alternativen transnationalen Einflussfaktoren (Auslandsaufenthalt; westliche Medien) um vier theoretisch relevante Kontrollvariablen: Bildungsniveau (Universitätsdiplom oder Doktorat), Position innerhalb der Staatsadministration (Abteilungsleiter oder -mitarbeiter), Geschlecht und Alter. Für das matching-Verfahren ersetze ich die fehlenden Datenwerte auf den unabhängigen Variablen durch multiple Imputationen $(m=5)$ (vgl. King et al. 2001: 49). ${ }^{4}$ Abbildung 1 stellt die Balance für die Kovariate vor und nach dem matchingVerfahren grafisch dar. Wie üblich bewerte ich die Ähnlichkeit zwischen den Verteilungen einer Kovariate innerhalb der Fokus- und Kontrollgruppe (»optimale Balance «) sowohl anhand der standardisierten Bias, das heißt dem Unterschied zwischen den Mittelwerten der Fokus- und Kontrollgruppe geteilt durch die zusammengefasste Standardabweichung (linkes Panel), als auch anhand der $p$-Werte (rechtes Panel) (vgl. Abadie 2002; Diamond/Sekhon 2008). Zur besseren Verständlichkeit sei darauf hingewiesen, dass für die binären Variablen die $p$-Werte durch einfache t-Tests und für die kontinuierliche Variable »Alter« durch mittels Bootstrap erzeugte Kolmogorov-Smirnov Tests kalkuliert (10.000 Bootstrap Samples) wurden. Ein standardisierter Bias innerhalb des Intervalls [-0.25; 0.25] gibt an, dass die jeweilige Variable gut ausbalanciert ist (Ho et al. 2007: 220). Das rechte Panel berichtet die $p$-Werte mit einem Grenzwert von 0.10 , um zu ermitteln, ob reale Unterschiede zwischen den beiden Gruppen weiterhin fortbestehen. Abbildung 1 zeigt, dass sich die beiden Gruppen von Staatsbeamten vor dem matching in der Tat bei einigen wenigen Variablen unterschieden. Mit dem matching-Verfahren konnten diese Unterschiede jedoch erfolgreich ausgeräumt werden: Die verbleibenden Mittelwertunterschiede sind minimal und rangieren deutlich zwischen $[-0.25 ; 0.25]$; einzig die Kovariate »Geschlecht« liegt mit einem Wert von -0.291 ( $p=.130)$ leicht über dem empfohlenen Richtwert. Auch liegen alle $p$-Werte über 0.10.

4 Für das Verfahren der multiplen Imputation verwende ich Amelia II's EMB-Algorithmus, siehe Honaker et al. (2007). Für das matching-Verfahren sowie die Regressionsanalysen kombiniere ich die fünf imputierten Datensätze auf Basis der kalkulierten Mittelwerte. 
Abbildung 1: Matching der Fokus- und Kontrollgruppe

\section{Standard. Mittelwertunterschiede}

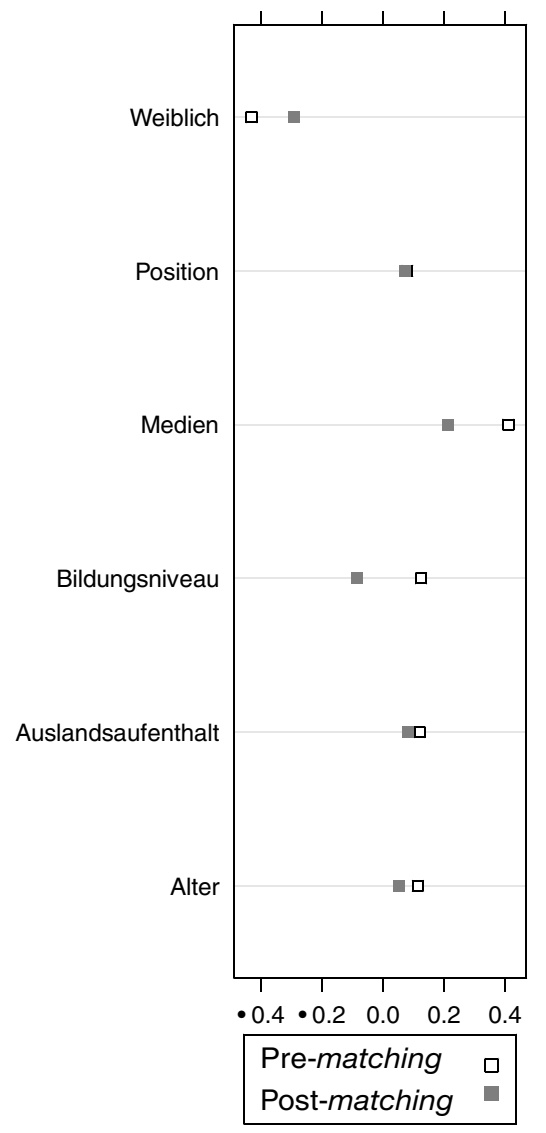

P-Werte

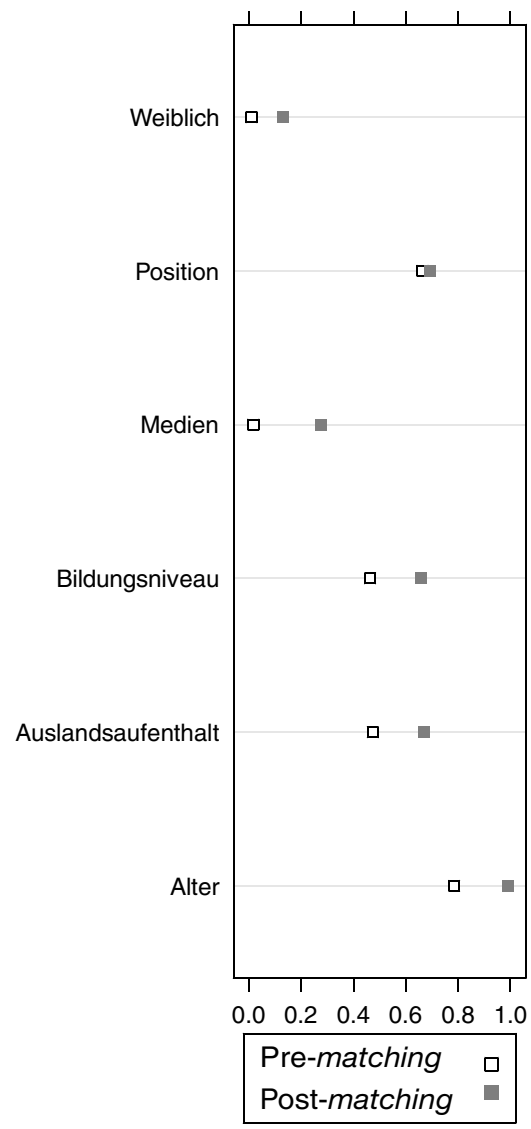

Linkes Panel: Unterschiede zwischen den Mittelwerten der Fokus- und der Kontrollgruppe, geteilt durch die zusammengefasste Standardabweichung; Rechtes Panel: p-Werte, erzeugt durch t-Tests für die binären Variablen und durch mittels Bootstrap erzeugte Kolmogorov-Smirnov Tests für die kontinuierliche Variable »Alter $« ;=150$ total.

Entscheidend ist nun vor diesem Hintergrund, ob das matching der Fokus- und Kontrollgruppe - wie von Bräuninger vermutet - einen Einfluss auf den geschätzten Effekt der Teilnahme an einem Twinning-Projekt auf die Einstellungen der befragten Staatsbeamten zu demokratischem Regieren hat. Tabelle 1 zeigt die Ergebnisse der Regressionsanalysen auf Basis des Datensatzes, den ich mithilfe des matching-Verfahrens erzeugt habe. Um einen besseren Vergleich zu gewährleisten, be- 
schränke ich mich ebenso wie Bräuninger auf die Darstellung der Ergebnisse zur abhängigen Kernvariablen »Demokratisches Regieren« und verwende zur Schätzung der Parameter ebenfalls einen einfachen OLS-Schätzer, auch wenn dies im Ergebnis keinen Unterschied machen sollte (siehe Hayashi 2000: 47). Im Unterschied zu Bräuningers Tabelle 1 (Bräuninger 2012: 93) zeigt meine Tabelle 1 keine Schätzung für Modell 5, da ich dieses in Tabelle A2 zu den Interaktionseffekten aufnehme. Tabelle 1 enthält dafür ein weiteres Modell 6 mit den zusätzlichen Kontrollvariablen, welche ich für das matching-Verfahren verwendete.

Tabelle 1: Regressionsergebnisse nach matching-Verfahren

\begin{tabular}{|c|c|c|c|c|c|}
\hline Items & Modell 1 & Modell 2 & Modell 3 & Modell 4 & Modell 6 \\
\hline (Konstante) & $\begin{array}{c}4.147 * * * \\
(.193)\end{array}$ & $\begin{array}{c}4.109 * * * \\
(.197)\end{array}$ & $\begin{array}{c}.153 * * * \\
(.192)\end{array}$ & $\begin{array}{c}4.112^{* * * *} \\
(.199)\end{array}$ & $\begin{array}{c}2.250 \\
(7.698)\end{array}$ \\
\hline Kooperation & $\begin{array}{l}.135 \\
(.085)\end{array}$ & $\begin{array}{l}.126 \\
(.085)\end{array}$ & $\begin{array}{l}-.046 \\
(.109)\end{array}$ & & $\begin{array}{l}-.082 \\
(.108)\end{array}$ \\
\hline Admin. Sozialisation & & $\begin{array}{l}-.033 \\
(.083)\end{array}$ & $\begin{array}{l}-.040 \\
(.081)\end{array}$ & $\begin{array}{l}-.051 \\
(.083)\end{array}$ & $\begin{array}{l}-.249^{*} \\
(.124)\end{array}$ \\
\hline Auslandsaufenthalt & & $\begin{array}{l}-.016 \\
(.082)\end{array}$ & $\begin{array}{l}-.257^{*} \\
(.128)\end{array}$ & $\begin{array}{l}-.012 \\
(.082)\end{array}$ & $\begin{array}{l}-.395 \\
(.789)\end{array}$ \\
\hline Ausl. Medien & & $\begin{array}{l}-.037 \\
(.145)\end{array}$ & $\begin{array}{l}-.043 \\
(.142)\end{array}$ & $\begin{array}{l}-.031 \\
(.146)\end{array}$ & $\begin{array}{c}-.478 \\
(3.669)\end{array}$ \\
\hline Andere Programme & & $\begin{array}{l}.221 * * \\
(.081)\end{array}$ & $\begin{array}{l}.213 * * \\
(.079)\end{array}$ & $\begin{array}{l}.221 * * \\
(.081)\end{array}$ & $\begin{array}{l}.209 * * \\
(.078)\end{array}$ \\
\hline Koop. x Auslandsauf. & & & $\begin{array}{l}. \mathbf{3 8 8} \text { * } \\
(.161)\end{array}$ & & $\begin{array}{l}.418^{*} \\
(.160)\end{array}$ \\
\hline Alter & & & & & $\begin{array}{l}.023 \\
(.036)\end{array}$ \\
\hline Weiblich & & & & & $\begin{array}{c}.422 \\
(3.314)\end{array}$ \\
\hline Bildungsniveau & & & & & $\begin{array}{l}-.025 \\
(.107)\end{array}$ \\
\hline Position & & & & & $\begin{array}{l}.072 \\
(.224)\end{array}$ \\
\hline Distanz & $\begin{array}{l}-.298 \\
(.338)\end{array}$ & $\begin{array}{l}-.334 \\
(.437)\end{array}$ & $\begin{array}{l}-.209 \\
(.429)\end{array}$ & $\begin{array}{l}-.201 \\
(.430)\end{array}$ & $\begin{array}{c}1.706 \\
(16.283)\end{array}$ \\
\hline Angepasstes $R^{2}$ & .007 & .045 & .094 & .032 & .127 \\
\hline Resid. sd. & .390 & .385 & .374 & .387 & .368 \\
\hline$N$ & 96 & 95 & 95 & 95 & 95 \\
\hline
\end{tabular}

Nichtstandardisierte Regressionskoeffizienten mit Standardfehlern in Klammern; Fälle mit fehlenden Werten listenweise ausgeschlossen; ${ }^{\circ} p \leq 05,{ }^{*} p \leq 01,{ }^{* *} p \leq 001,{ }^{*} * * \leq 000$.

Die in Tabelle 1 gezeigten Regressionsergebnisse sind im Wesentlichen identisch mit den Ergebnissen, die mit dem Originaldatensatz ohne matching-Verfahren erzeugt wurden. Die Ergebnisse meiner Studie stützen »das Argument vom demokra- 
tischen Potenzial funktionaler Kooperation. Sie zeigen jedoch auch auf, dass allein die Teilnahme an einem Twinning-Projekt die Einstellungen der involvierten Beamten nicht signifikant prägt (Modell 1). Der Koeffizient für Kooperation ist zwar positiv, aber nicht signifikant« (Freyburg 2011: 24). Auch nach dem matching-Verfahren finde ich keinen unabhängigen demokratisierenden Effekt von Auslandsaufenthalten und der Nutzung westlicher Medien. Dafür zeigt sich jedoch weiterhin ein robuster positiver und signifikanter Effekt der Teilnahme an Politikreformprogrammen im Allgemeinen. Wie im Folgenden noch besprochen wird, ist auch in Tabelle 1 der Koeffizient des Interaktionsterms von Kooperation und Auslandsaufenthalt in Modell 3 (und auch Modell 6, das für zusätzliche Einflussfaktoren kontrolliert) positiv und statistisch signifikant. Zusammenfassend kann damit festgehalten werden, dass selbst wenn ich mithilfe eines matching-Verfahrens für ein etwaiges Selektionsproblem kontrolliere, die Ergebnisse der Regressionsanalyse robust bleiben.

\subsection{Interpretation des Interaktionseffektes}

Während meine Studie mit der systematischen Untersuchung des demokratisierenden Potenzials transgouvernementaler Netzwerke empirisch-analytisches Neuland betritt, ist die transnationale Diffusion demokratischer Normen ein gut etabliertes Forschungsfeld. ${ }^{5}$ Existierende Studien identifizieren insbesondere zwei Formen der grenzüberschreitenden Interaktion als bedeutende Transmitter demokratischer Prinzipien und Praktiken in nichtdemokratische Staaten: Auslandsaufenthalte zu Ausbildungs- oder beruflichen Zwecken in etablierten Demokratien und westliche Medien zur politischen Information (siehe Freyburg 2011: 12). In der ursprünglichen Studie interessierte mich, inwieweit diese alternativen transnationalen Faktoren den Effekt transgouvernementaler Netzwerke auf die Einstellungen zu demokratischem Regieren von Staatsbeamten beeinflussen. Ein direkter, unabhängiger Sozialisierungseffekt von Auslandsaufenthalt bzw. ausländischen Medien lag außerhalb meines Erkenntnisinteresses. Konkret formulierte ich die Erwartung, dass »Staatsbeamte« (Freyburg 2011: 12) (d.h. nicht ausschließlich »Projektteilnehmer«, Bräuninger 2012: 94), die bereits alternativen, in der Literatur als demokratisierend identifizierten Einflüssen ausgesetzt waren und dann an einem Twinning-Projekt teilnehmen, positivere Einstellungen zu demokratischem Regieren haben als diejenigen Beamte, die nicht in ein Twinning-Projekt involviert waren. Damit fokussierten sich die Hypothesen $2 \mathrm{a}$ und $2 \mathrm{~b}$ allein auf den potenziell begünstigenden Effekt der alternativen transnationalen Einflussfaktoren auf die erwartete demokratisierende Wirkung von transgouvernementalen Netzwerken.

Die Erwartung eines solchen Effektes früherer Erfahrungen auf das Demokratisierungspotenzial der Netzwerke formulierte ich auf der Basis insbesondere zweier

5 Siehe zum Beispiel Cederman/Gleditsch (2004); Levitsky/Way (2005); Simmons et al. (2006); Elkink (2011). 
- auf den ersten Blick in der Tat widersprüchlicher - Befunde aus der Sozialpsychologie. Bräuninger gewann daher den Eindruck, das theoretische Argument, das Hypothese 2a und 2b zugrunde liegt, sei »fehlerhaft « (Bräuninger 2012: 89). Im Folgenden erläutere ich die theoretische Herleitung meines Argumentes etwas ausführlicher. Der primacy effect prognostiziert, dass Erfahrungen einen größeren Einfluss haben, wenn »a person has few relevant prior experiences« (Hooghe 2005: 866). Der repetition effect postuliert, dass wiederholte Erfahrungen besonders einflussreich sind, da sie die Möglichkeit bieten »to elaborate cognitively upon them and to realize their cogency and favorable implications « (Cacioppo/Petty 1979: 105). Eine nähere Beschäftigung mit der Literatur macht deutlich, dass beide Effekte auf denselben Mechanismus einer Sozialisierung durch Erfahrungen hindeuten. In dem von Bräuninger (und auch mir) zitierten Beitrag von Hooghe geht es um die Präferenzen von höheren Kommissionsbeamten im Hinblick auf Europäisches Regieren. Hooghe formuliert darin die Erwartung, dass nationale Beamte »are [the] more susceptible to [European] socialization «, desto früher sie auf ihrem Berufsweg nach Brüssel gesandt werden. Der Grund für diese Erwartung ist, dass jüngere Rekruten weniger durch Erfahrungen in nationalen Bürokratien geprägt wurden und somit »may have [not yet] internalized the value of preserving national sovereignty« (Hooghe 2001: 101). Kurzum, und übertragen auf meine Studie zur demokratischen Sozialisation von arabischen Beamten in transgouvernementalen Netzwerken, entscheidend ist weniger »das (biologische) Alter des Individuums sowie die Menge seiner bisherigen Erfahrungen« (Bräuninger 2012: 88). Vielmehr kommt es darauf an, was (wie häufig) erfahren wurde. Die in meinem Beitrag angegeben Referenzen »Johnston 2001: 497 « und »Checkel 2001: 563« (Freyburg 2011: 13) stützen diese Lesart der psychologischen Literatur. So heißt es beispielsweise bei Jeffrey T. Checkel: »[a]rgumentative persuasion is more likely to be effective when the persuadee has few prior, ingrained beliefs that are inconsistent with the persuader's message « (Checkel 2001: 563, meine Hervorh.). Folglich gilt: Je weniger Erfahrungen Beamte bereits mit (autoritärem) Regieren gemacht haben, desto wahrscheinlicher ist eine effektive Sozialisation in demokratischem Regieren zu einem späteren Zeitpunkt, ${ }^{6}$ bzw. je mehr Erfahrungen sie bereits mit demokratischem Regieren gemacht haben, desto wahrscheinlicher ist eine positive Beeinflussung ihrer Einstellungen zu demokratischem Regieren zu einem späteren Zeitpunkt. ${ }^{7}$ Auf Grundlage dieser Überlegungen formulierte ich die Erwartung, dass »Staatsbeamte, die bereits Erfahrungen mit demokratischem Regieren gemacht haben, eher dazu geneigt sind, ihre Einstellungen zu ändern, wenn sie erneut in Kontakt mit demokratischem Regieren kommen« (Freyburg 2011: 13).

Zur empirischen Überprüfung der Hypothesen $2 \mathrm{a}$ und $2 \mathrm{~b}$ führte ich in meiner ursprünglichen Studie die Kreuzprodukte der binären Variablen »Kooperation« und

6 Dies ist im Übrigen auch der Grund, warum ich in meiner Arbeit für administrative Sozialisation kontrolliere (siehe Freyburg 2011: 22).

7 Anders ausgedrückt: Während in der ersten Aussage »few« in Hooghes Zitat die Bedeutung von >nur wenige annimmt, kann es in der zweiten Aussage mit >mindestens einige übersetzt werden. Ich danke Solveig Richter für diesen Hinweis. 
den alternativen transnationalen Einflussfaktoren ebenfalls als binäre Variablen einzeln in die Regressionsanalyse ein. Die in Tabelle 3 meines Beitrages abgebildeten Ergebnisse $^{8}$ zeigen nicht nur einen positiven und statistisch signifikanten Koeffizienten des statistischen Interaktionsterms, sondern auch einen »signifikanten marginalen Effekt der Kooperation für Beamte mit Auslandserfahrung« (Bräuninger 2012: 94). Dieser Effekt bleibt signifikant, wenn man für einen potenziellen Selektionseffekt mithilfe eines matching-Verfahrens kontrolliert, wie hier aufgezeigt.

\section{Tabelle 2: Deskriptive Statistik zum Interaktionseffekt »Kooperation x Auslandsauf- enthalt«}

Teilnahme an einem Twinning-Projekt (»Kooperation«)

Nein

$\mathrm{Ja}$

\begin{tabular}{llllll}
\hline & Nein & $4.10(.363)$ & & $4.02(.313)$ & \\
\cline { 2 - 6 } Auslandsaufenthalt & & (1) & & (2) \\
\cline { 2 - 6 } & $\mathrm{Ja}$ & $3.88(.492)$ & (3) & $4.18(.346)$ & (4) \\
\hline
\end{tabular}

Arithmetisches Mittel der Einstellung zu demokratischem Regieren mit Standardabweichung in Klammen; $N(1)=31, N(2)=32, N(3)=20, N(4)=26$; Werte rangieren von 1 (»nichtdemokratisch «) zu 5 (»demokratisch $\ll$ ).

Tabelle 2 zeigt die deskriptive Statistik der Interaktion »Kooperation x Auslandsaufenthalt«. Bräuningers Abbildung 1 (Bräuninger 2012: 95) stellt diese Vier-Felder-Matrix grafisch mit (vermutlich 95\%) Konfidenzintervallen dar. ${ }^{9}$ Auf Basis dieser Grafik schlussfolgert Bräuninger, dass es den postulierten Einstellungsunterschied nicht gibt. Zu diesem Schluss kommt er, indem er die Einstellungen von Beamten ohne Auslandserfahrung mit den Einstellungen von Beamten mit entsprechender Erfahrung »im Mittel« vergleicht. Entscheidend ist für ihn dabei die Frage eines signifikanten Einstellungsunterschieds »zwischen Beamten ohne Auslands- und Kooperationserfahrung und Beamtinnen, welche beide Erfahrungen haben « (Bräuniger 2012: 94), also zwischen den Gruppen 1 und 4 in Tabelle 2. Dies ist eine interessante und legitime Frage; die mangelnde empirische Evidenz verlangt zudem klar nach weiterer Forschung zu der Mikrofundierung transnationaler Diffusionsprozesse. Allein, sie ist nicht Gegenstand der Hypothese 2a.

8 »Tabelle 3 (Freyburg 2011: 25) zeigt nur die signifikanten Interaktionseffekte« (Freyburg 2011: 24). Aus diesem Grund enthält sie nur Modell 3a mit dem Interaktionsterm »Kooperation x Auslandsaufenthalt « (Hypothese 2a) und kein Modell mit dem Interaktionsterm »Kooperation x Medien« (Hypothese 2b). Tabelle A2 im Anhang stellt alle Modelle mit Interaktionstermen dar, die ich aus Gründen der besseren Vergleichbarkeit ebenfalls mit einem einfachen OLS-Schätzer und dem originären Datensatz rechnete.

9 Der erste Graph von links (»weder noch«) bildet Feld $1 \mathrm{ab}$, der zweite Graph (»nur Kooperation«) Feld 2, der dritte Graph dementsprechend Feld 3 und der letzte Graph (»beides«) Feld 4. Ich danke Philipp Rehm für seine Vorschläge zur Überprüfung des Interaktionseffektes und Julian Wucherpfennig für seine Unterstützung bei Abbildung 2. 
Hypothese 2a kann dann (und nur dann) bestätigt werden, wenn die Teilnahme an einem Twinning-Projekt allein bei vorherigem Auslandsaufenthalt einen Effekt hat. Konkret heißt dies: Es muss einen signifikanten Unterschied in dem Niveau der Einstellungen zu demokratischem Regieren zwischen den Gruppen 4 und 3, aber nicht zwischen den Gruppen 1 und 2 geben. Dies entspricht auch dem angewandten quasi-experimentellen Design mit Gruppe 4 als Fokus- und Gruppe 3 als Kontrollgruppe. Ich nehme Bräuningers Skepsis zum Anlass, diese beiden relevanten Implikationen der Hypothese 2a mithilfe von dummy-Variablen statistisch zu testen. Hierzu generiere ich pro Gruppe eine dichotome Variable (D1, D2, D3, D4) und führe drei der vier Variablen (D2, D3, D4) in die Regressionsanalyse ein (Modell 8 in Tabelle A2). Anschließend teste ich mithilfe eines Wald-Tests die Nullhypothesen für D4-D3 und D2-D1. Nach diesem Koeffiziententest kann die erste Nullhypothese abgelehnt (D4>D3; $\left.p=.013 ; \mathrm{df}=1 ; x^{2}=6.139\right)$ und die zweite Nullhypothese (D2=0; $\left.p=.344 ; \mathrm{df}=1 ; x^{2}=.894\right)$ angenommen werden. Damit gibt es einen signifikanten Unterschied in den Einstellungen der Gruppen 3 und 4, aber nicht der Gruppen 1 und 2. Abbildung 2 reduziert Bräuningers Abbildung 1 (Bräuninger 2012: 95) auf den rechten Graphen, also den signifikanten Einstellungsunterschied zwischen Beamten mit Auslandserfahrung und Beamten mit Auslands- und Kooperationserfahrung. ${ }^{10}$ Ich schätze den marginalen Effekt dieses Interaktionsterms aufgrund des kleinen Samples mit 90\% Konfidenzintervall basierend auf Simulationen $(\mathrm{N}=1000)$. Abbildung 2 zeigt deutlich, dass sich die Einstellung von Beamten zur Angemessenheit von demokratischem Regieren mit Auslandserfahrung von 3.87 auf 4.14 statistisch signifikant auf einer Skala von 1 (»nichtdemokratisch «) bis 5 (»demokratisch«) erhöht, wenn sie zu einem späteren Zeitpunkt an einem Twinning-Projekt teilnehmen. Dies entspricht dem positiven, signifikanten marginalen Effekt von .272 ( $p=.015)$. Damit findet sich in den empirischen Daten eindeutig Evidenz für das Argument, welches Hypothese 2a zugrunde liegt.

10 Selbst wenn man umgekehrt argumentieren und Twinning-Teilnehmer mit und ohne Auslandserfahrung vergleichen möchte, stützt Bräuningers Abbildung 1 (Bräuninger 2012: 95) das Argument eines begünstigten Einfluss von Auslandsaufenthalt auf einen Sozialisierungseffekt von Kooperation. Das Niveau der Einstellungen von Teilnehmern mit Auslandserfahrung (Graph rechts außen) ist - wenn auch nicht signifikant - höher als das Niveau der Einstellungen von Teilnehmern ohne Auslandserfahrung (zweiter Graph von links). 
Abbildung 2: Interaktionseffekt für Kooperation und Auslandsaufenthalt (Hypothese 2a)

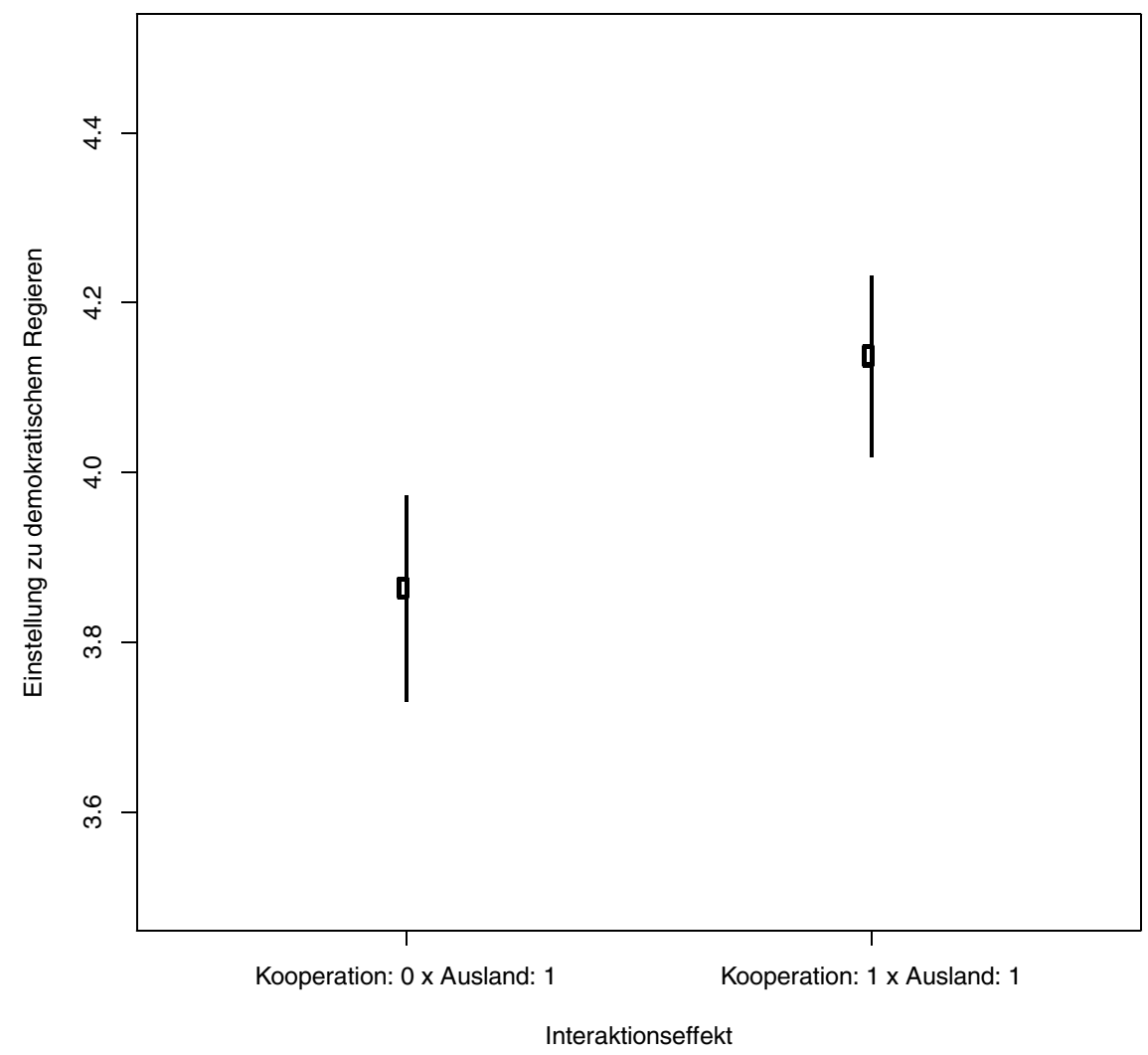

Mittelwertunterschiede für Beamte mit Auslandserfahrung (links) und Beamte mit Auslands- und Kooperationserfahrung (rechts) mit 90\% Konfidenzintervall basierend auf Simulationen ( $N=1000)$.

In meiner Arbeit argumentiere ich, dass dieser Befund eines konditionalen Effekts der Netzwerke in erster Linie bedeutet, »dass ein vorheriger direkter Kontakt mit demokratischen Prinzipien - unabhängig von ihrer Bewertung - notwendig zu sein scheint, damit ein späterer zielgerichteter Austausch über diese Prinzipien an bereits vorhandene $[\ldots]$ mentale Wissensstrukturen anknüpfen $[\ldots]$ kann« (Freyburg 2011: 26). Bräuninger argumentiert, es handele sich hier nicht um ein »>Anknüpfen an frühere Erfahrungen«, sondern entweder um eine »Kompensation früherer negativer Erfahrungen« (Bräuninger 2012: 92) oder um einen Selektionseffekt »bei der Rekrutierung der Beamten« (Bräuninger 2012: 94). Bei einem nicht signifikanten Koeffizienten von -.052 vor und von -.016 $(p=.843)$ nach dem matching-Verfahren, würde ich nicht davon sprechen wollen, dass ein Auslandsaufenthalt tat- 
sächlich einen negativen Wandel der Einstellungen zu demokratischem Regieren bewirkt (Modell 2 in Bräuningers Tabelle 1 bzw. in obiger Tabelle 1). Hingegen scheint es, dass Beamte bereits eine Vorstellung von demokratischen Verfahren haben müssen, damit Aktivitäten der Twinning-Programme eine demokratisierende Wirkung haben. Ich stimme Bräuninger zu, dass es sich bei Nutzern westlicher Medien und mobilen Studenten um eine selbst-selektierende Gruppe handeln mag. Es kann jedoch erwartet werden, dass eine solche Selbstselektion die Schätzungen eines demokratisierenden Effektes in konträre Richtungen beeinflusst: verstärkend im Falle Mediennutzung und abschwächend im Falle Auslandsaufenthalt. So ist denkbar, dass sich vor allem Studenten aus regimetreuen Familien mit guten Verbindungen zur politischen Elite und daher eher negativen Einstellungen zu demokratischem Regieren einen Auslandsaufenthalt erlauben können. Westliche Medien wiederum könnten besonders für Staatsbeamte attraktiv sein, die externen Einflüssen gegenüber bereits aufgeschlossener sind und sich mehr für Ereignisse im demokratischen Ausland interessieren. Vor diesem Hintergrund sollten wir folglich einen stärkeren Effekt von westlichen Medien auf die Einstellungen zu demokratischem Regieren als von Auslandsaufenthalten beobachten. Abbildung 3 zeigt, dass dies nicht der Fall ist. Die dargestellten boxplots bilden die Mittelwerte der Einstellungen zu demokratischem Regieren (50\% der erhobenen Daten) ab, wobei die vertikalen Linien die Verteilung der Werte anzeigen; die horizontalen Linien geben den Median an. Das durchschnittliche Niveau der Einstellungen beider treatment-Gruppen ist fast identisch mit einem arithmetischen Mittel von 4.49 und einem Median von 4.10 für Auslandsaufenthalt $(N=46)$ und einem arithmetischen Mittel von 4.04 und einem Median von 4.11 für Medien $(N=84)$. Nichts deutet darauf hin, dass Beamte mit überwiegend negativen Einstellungen $\mathrm{zu}$ demokratischem Regieren im Ausland studierten und Beamte mit überwiegend positiven Einstellungen westliche Medien zur politischen Information nutzen. Kurzum, Hypothese $2 \mathrm{a}$ zu einem positiven Einfluss eines vorherigen Auslandsaufenthaltes auf einen sozialisierenden Effekt von Netzwerken ist theoretisch gerechtfertigt und kann empirisch bestätigt werden. 
Abbildung 3: Einstellungen zu demokratischem Regieren

Auslandsaufenthalt

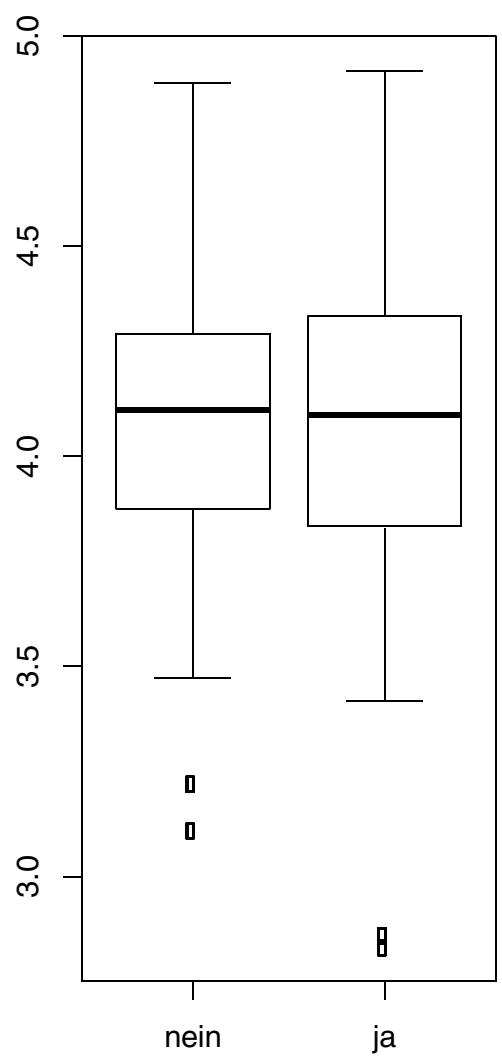

Medien

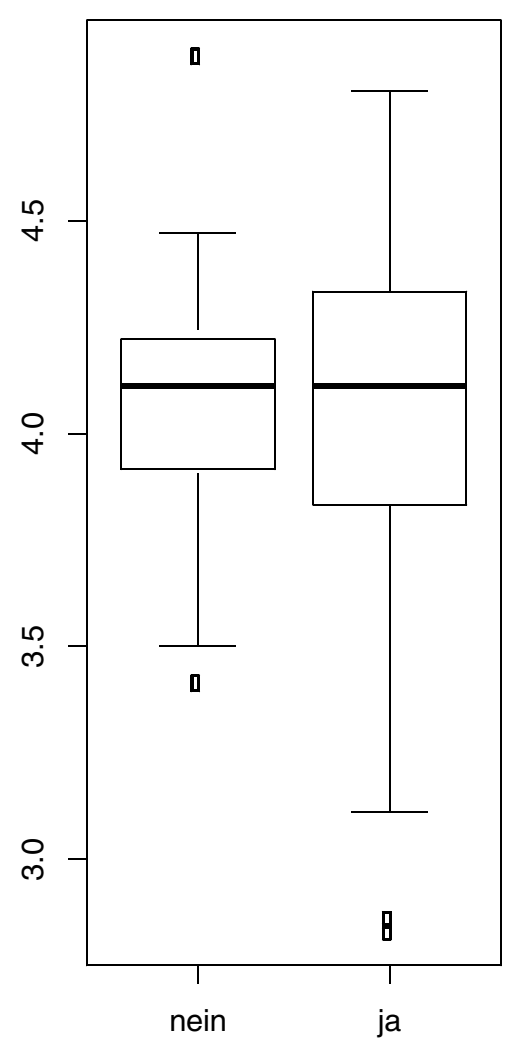

Boxplots für Einstellungen zu demokratischem Regieren von Beamten, die (nicht) im demokratischen Ausland waren (linkes Panel) bzw. westliche Medien (nicht) zur politischen Information nutzen (rechtes Panel). Werte rangieren von 1 (»nichtdemokratisch «) zu 5 (»demokratisch «); $N=150$, Fälle mit fehlenden Werten listenweise ausgeschlossen; »nein« = Kontrollgruppe, »ja» = Treatment-Gruppe.

\subsection{Vergleich einzelner Twinning-Projekte}

Sind bestimmte Twinning-Projekte geeigneter als andere, um teilnehmende Staatsbeamte in demokratischem Regieren zu sozialisieren? Wenn ja, welche spezifischen Eigenschaften könnten dieses unterschiedliche Demokratisierungspotenzial erklären? $\mathrm{Zu}$ diesem Zweck verglich ich in meinem Beitrag vier Twinning-Projekte, die ich auf Basis der Politisierung des Politikbereiches auswählte (Freyburg 2011: 26-27). Ich danke Thomas Bräuninger für seinen zusätzlichen Robustheitstest meiner Analysen durch die gleichzeitige Schätzung der demokratisierenden Effekte der 
individuellen Projekte in einem Modell. Einer der Hauptbefunde meiner Regressionsanalysen zu den individuellen Projekten sind die sektorspezifischen Effekte auf die Einstellungen zu den einzelnen Dimensionen von demokratischem Regieren, d.h. Partizipation, Transparenz und Verantwortlichkeit (Freyburg 2011: 27-28). Leider beschränkt sich Bräuninger (2012: 95) auf die »übergeordnete Dimension des demokratischen Regierens« als abhängige Variable sowie auf ein Set an Kovariaten, das keine projektspezifischen Faktoren enthält. Auch rechnet Bräuninger die Interaktionsmodelle, welche eine Interpretation der konstitutiven Elemente eines Interaktionsterms als unabhängigen oder durchschnittlichen Effekt nicht erlauben (vgl. Brambor et al. 2006: 71). Im Rahmen dieser Modelle stimmen seine Ergebnisse im Wesentlichen mit meinen überein (vgl. Tabellen IVa-d; Freyburg 2011: 40-46). Seine konträre Einschätzung des Demokratisierungspotenzials der Projekte zu Umwelt und Wettbewerb und seine sich daraus ergebende Schlussfolgerung, dass die Politisierung des Politikbereiches nicht den erwarteten abschwächenden Effekt hat, beruht ausschließlich auf den durch bootstrap erhaltenen Standardfehlern (Bräuninger 2012: 98). Die bootstrap-Methode ist ein resampling-Verfahren zur Ableitung von robusten Schätzwerten wie Standardfehlern aus dem ursprünglichen Datensatz. Diese Methode hat den Vorteil, dass sie nicht auf theoretischen Modellen basiert und daher keine genauen Voraussetzungen über die Verteilung der Beobachtungen getroffen werden müssen. Die einzige Voraussetzung, die beim bootstrap erfüllt sein muss, ist, dass die Stichprobe repräsentativ für die Population ist. Wenn ein Sample unter einem Selektionsbias leiden könnte - wie Bräuninger im vorliegenden Fall vermutet - sollte ein bootstrap-Verfahren nicht angewandt werden. Das Verfahren hat zudem auch den Effekt, dass Standardfehler bei kleinen Fallzahlen nach oben gehen. Diese modellbedingte Erhöhung der Standardfehler führt zu Bräuningers Zurückweisung des postulierten Effekts.

\section{Schlussbemerkung}

Inwiefern können Demokratisierungsprozesse in autoritären Regimen von außen gefördert werden? In meinem Beitrag untersuchte ich subtile Prozesse der Demokratisierung, welche als Nebeneffekt externer Aktivitäten entstehen können, die nicht primär der Demokratieförderung dienen. Konkret interessierte mich die Frage, inwiefern und unter welchen Bedingungen die Einstellung zu demokratischem Regieren von staatlichen Beamten, die Herrschaft im Alltag ausüben, durch externe Einflüsse wie interadministrative Kooperationsprogramme positiv beeinflusst werden können. Damit verbindet meine Studie eine Untersuchung der Mikrofundierung des Diffusionsargumentes mit einer Analyse von Sozialisierungsprozessen im transnationalen Raum. Sozialisierung ist nicht nur ein Kernelement der meisten Theorien über die soziale Wirklichkeit, es ist auch ein faszinierendes Forschungsgebiet, das Wissenschaftler vor viele konzeptionelle und methodologische Herausforderungen stellt (vgl. Pollack 1998; Beyers 2010). In meiner Forschung stelle ich mich diesen Herausforderungen und versuche neue Wege zu gehen, um transnatio- 
nale Sozialisierungsprozesse und -effekte auf Mikroebene zu untersuchen. Konkret versuchte ich in meinem ZIB-Beitrag, Prozesse des Normentransfers durch transgouvernementale Kooperation auf der Ebene staatlicher Administrationen zu erfassen und demokratische Spuren empirisch nachzuweisen, die externe Einflüsse bei innerstaatlichen Akteuren in autoritären Kontexten hinterlassen haben können, auch wenn sie in beobachtbarem Verhalten (noch?) nicht sichtbar sind. Unter bestimmten Bedingungen können auch vermeintliche Wüsten sich als fruchtbar erweisen. Wir müssen nur genau hinschauen. Ich danke Thomas Bräuninger für seinen Beitrag zur kritischen Hinterfragung und Verfeinerung meiner Analysen.

\section{Literatur}

Abadie, Alberto 2002: Bootstrap Tests for Distributional Treatment Effects in Instrumental Variable Models, in: Journal of the American Statistical Association 97: 457, 284-292.

Agresti, Alan/Finlay, Barbara 2009: Statistical Methods for the Social Sciences, 4. Auflage, New Jersey, NJ.

Beyers, Jan 2010: Conceptual and Methodological Challenges in the Study of European Socialization, in: Journal of European Public Policy 17: 6, 909-920.

Bräuninger, Thomas 2012: Fruchtbarer Boden oder doch nur Wüste? Eine Replik auf Tina Freyburgs Beitrag zur Demokratisierung durch Zusammenarbeit, in: Zeitschrift für Internationale Beziehungen 19: 1, 85-101.

Brambor, Thomas/Clark, William R./Golder, Matt 2006: Understanding Interaction Models: Improving Empirical Analyses, in: Political Analysis 14: 1, 63-82.

Cacioppo, John T./Petty, Richard E. 1979: Effects of Message Repetition and Position on Cognitive Response, Recall, and Persuasion, in: Journal of Personality and Social Psychology 37: 1, 97-109.

Campbell, Donal T./Stanley, Julian C. 1966: Experimental and Quasi-Experimental Designs for Research, Chicago, IL.

Cederman, Lars-Erik/Gleditsch, Kristian S. 2004: Conquest and Regime Change: An Evolutionary Model of the Spread of Democratic Peace, in: International Studies Quarterly 48: 3, 603-629.

Checkel, Jeffrey T. 2001: Why Comply? Social Learning and European Identity Change, in: International Organization 55: 3, 553-588.

Diamond, Alexis/Sekhon, Jasjeet S. 2008: Genetic Matching for Estmating Causal Effects: A General Multivariate Matching Method for Achieving Balance in Observational Studies (Version 11/14/2010, UC Berkeley: Institute of Governmental Studies).

Elkink, Johan A. 2011: The International Diffusion of Democracy, in: Comparative Political Studies 44: 2, 1651-1674.

Freyburg, Tina 2011: Demokratisierung durch Zusammenarbeit? Funktionale Kooperation mit autoritären Regimen und Sozialisation in demokratischem Regieren, in: Zeitschrift für Internationale Beziehungen 18: 1, 5-46.

Halaby, Charles N. 2004: Panel Models in Sociological Research: Theory into Practice, in: Annual Review of Sociology 30: 1, 507-44.

Hayashi, Fumio 2000: Econometrics, Princeton, NJ.

Ho, Daniel E./Imai, Kosuke/King, Gary/Stuart, Elizabeth A. 2011: MatchIt: Nonparametric Preprocessing for Parametric Causal Inference, in: http://gking.harvard.edu/matchit; 28.02.2012.

Hooghe, Liesbet 2001: The European Commission and the Integration of Europe: Images of Governance, Cambridge. 
Hooghe, Liesbet 2005: Several Roads Lead to International Norms, but Few via International Socialization: A Case Study of the European Commission, in: International Organization 59: 4, 861-898.

Honaker, James/ King, Gary/Blackwell, Matthew 2007: Amelia II: A Program for Missing Data, in: http://gking.harvard.edu/amelia/; 28.02.2012.

Johnston, Alaistair Iain 2001: Treating International Institutions as Social Environments, in: International Studies Quarterly 45: 4, 487-515.

Keohane, Robert O./Nye, Joseph S. 1974: Transgovernmental Relations and International Organizations, in: World Politics 26: 1, 39-62.

Kerr, Henry H. 1973: Changing Attitudes through International Participation: European Parlamentarians and Integration, in: International Organization 27: 1, 45-83.

King, Gary/Honaker, James/Joseph, Anne/Scheve, Kenneth 2001: Analyzing Incomplete Political Science Data: An Alternative Algorithm for Multiple Imputation, in: American Political Science Review 95: 1, 49-69.

Levitsky, Steven/Way, Lucan A. 2005: International Linkage and Democratization, in: Journal of Democracy 13: 2, 51-65.

Pollack, Mark A. 1998: Constructivism, Social Psychology, and Elite Attitude Change: Lessons from an Exhausted Research Programm (Manuskript für die 11. Konferenz der Europawissenschaftler, Baltimore, MD, 26.02.-01.03. 1998).

Raustiala, Kai 2002: The Architecture of International and the Future of International Law, in: Virginia Journal of International Law 43: 1, 1-92.

Simmons, Beth A./Dobbin, Frank/Garrett, Geoffrey 2006: Introduction: The International Diffusion of Liberalism, in: International Organization 60: 4, 781-810.

Slaughter, Anne-Marie 2004: A New World Order, Princeton, NJ.

Anhang

Tabelle A1: Matching der Fokus- und Kontrollgruppe

\begin{tabular}{|c|c|c|c|c|c|c|c|c|c|}
\hline & \multicolumn{3}{|c|}{ Pre-matching } & \multicolumn{3}{|c|}{ Post-matching } & \multirow{2}{*}{$\begin{array}{c}\% \mathrm{SB} \\
\text { Reduk- } \\
\text { tion }\end{array}$} & \multirow{2}{*}{$\begin{array}{c}\text { Pre } \\
p- \\
\text { Wert }\end{array}$} & \multirow{2}{*}{$\begin{array}{c}\text { Post } \\
p \text { - } \\
\text { Wert }\end{array}$} \\
\hline & $\begin{array}{c}\text { Fokus } \\
\varnothing\end{array}$ & $\begin{array}{c}\text { Kon- } \\
\text { trol } \\
\varnothing\end{array}$ & SB & $\begin{array}{c}\text { Fokus } \\
\varnothing\end{array}$ & $\begin{array}{c}\text { Kon- } \\
\text { trol } \\
\varnothing\end{array}$ & SB & & & \\
\hline Auslandsaufenthalt & .459 & .400 & .119 & .459 & .419 & .080 & 32.54 & .474 & .668 \\
\hline Medien & .871 & .708 & .410 & .871 & .791 & .213 & 47.91 & .018 & .275 \\
\hline Bildungsniveau & 1.66 & 1.60 & .124 & 1.66 & 1.7 & -.085 & 168.72 & .464 & .659 \\
\hline Alter & 41 & 40.1 & .114 & 41 & 40.6 & .053 & 53.86 & .783 & .991 \\
\hline Weiblich & .259 & .462 & -.431 & .259 & .395 & -.291 & 32.50 & .011 & .130 \\
\hline Position & 4.67 & 4.63 & .079 & 4.67 & 4.63 & .070 & 11.46 & .664 & .692 \\
\hline$N$ & 85 & 65 & & 85 & $43(7)$ & & & & \\
\hline
\end{tabular}

Fokus $\varnothing=$ Arithmetisches Mittel der Fokusgruppe; Kontrol $\varnothing=$ Arithmetisches Mittel der Kontrollgruppe. SB (Standardisierter Bias) gibt den Unterschied zwischen den Mittelwerten der Fokus- und der Kontrollgruppe an, geteilt durch die zusammengefasste Standardabweichung. \% SB Reduktion =Verbesserung der Balance nach dem matching-Verfahren in Prozent. Die p-Werte wurden für die binären Variablen durch t-Tests und für die kontinuierliche Variable "Alter « durch mittels Bootstrap erzeugte Kolmogorov-Smirnov Tests kalkuliert (10.000 Bootstrap Samples). N=150 total; verworfene Beobachtungen in Klammern. 


\section{Forum}

Tabelle A2: Ergebnisse der Regressionsanalysen mit Interaktionstermen/DummyVariablen

\begin{tabular}{|c|c|c|c|c|}
\hline Items & Modell 3a & Modell 5 & Modell 7 & Model 8 \\
\hline (Konstante) & $4.073 * * *(.093)$ & $4.064 * * *(.101)$ & $4.009 * * *(.101)$ & $4.073 * * *(.093)$ \\
\hline Kooperation & $-.092(.098)$ & $-.054(.184)$ & $.081(.181)$ & \\
\hline Administrative Sozialisation & $-.042(.077)$ & $-.045(.078)$ & $-.037(.080)$ & $-.042(.077)$ \\
\hline Auslandsaufenthalt & $-.251 *(.108)$ & $-.253 *(.109)$ & $-.053(.076)$ & \\
\hline Ausländische Medien & $-.055(.096)$ & $-.037(.122)$ & $-.079(.124)$ & $-.055(.096)$ \\
\hline Andere Programme & $.218 * *(.072)$ & $.217 * *(.073)$ & $.216 * *(.075)$ & $.218 * *(.072)$ \\
\hline $\begin{array}{l}\text { Kooperation x 1: Auslands- } \\
\text { aufenthalt }\end{array}$ & $.364 *(.145)$ & $.366 *(.146)$ & & \\
\hline $\begin{array}{l}\text { Kooperation x 1: } \\
\text { Ausländische Medien }\end{array}$ & & $-.048(.196)$ & $-.015(.201)$ & \\
\hline $\mathrm{D} 2$ & & & & $-.092(.098)$ \\
\hline D3 & & & & $-.251 *(.107)$ \\
\hline D4 & & & & $.021(.101)$ \\
\hline Angepasstes $R^{2}$ & .099 & .090 & .040 & .099 \\
\hline Resid. sd. & .358 & .360 & .340 & .358 \\
\hline
\end{tabular}

Regressionskoeffizienten sind nicht standardisiert; Standardfehler in Klammern; Fälle mit fehlenden Werten listenweise ausgeschlossen; ${ }^{\circ} p \leq .05,{ }^{*} p \leq .01,{ }^{*} *_{p} \leq .001, *^{*} * \leq \leq 000 ; N=103$. 